\title{
Antitumor Effect of Fever Range Whole Body Hyperthermia with Curcumin in Breast Cancer-induced Mice
}

\author{
Hanim Saim, Maheza Irna Mohamad Salim, Khairunadwa Jemon
}

\begin{abstract}
Breast cancer is a complex and heterogeneous disease and also one of the major cancer types among female worldwide. Fever range whole-body hyperthermia and curcumin as a single treatment have been tested against breast cancer and showed some promising anti-tumor effect. However, their combination as an effective anti- tumor treatment against breast cancer has never been explored. The effects of combined wholebody fever range hyperthermia and curcumin on tumor growth was examined in this study. Mice were inoculated with EMT6 cells subcutaneously and allocated to 4 treatment groups: (i) control (control), (ii) curcumin (50mg/kg bodyweight), (iii) twice fever range whole-body hyperthermia $39.0^{\circ} \mathrm{C}( \pm 0.5)$ for 15 minutes, (iv) a combination of curcumin (50mg/kg bodyweight) and twice fever range whole-body hyperthermia $39.0^{\circ} \mathrm{C}( \pm 0.5)$ for 15 minutes. Following treatment, mice body weight and tumor volume were measured. The greatest tumor growth inhibition exhibited in combination treatment $(68.45 \%, p<0.05)$ and showed no general toxicity. As conclusion, the combination treatment can be a potential anti-tumor treatment of breast cancer.
\end{abstract}

Index Terms: breast cancer, curcumin, fever-range whole body hyperthermia (FRWBH).

\section{INTRODUCTION}

Breast cancer is the most common cancer occurring among women throughout the world. In Malaysia, breast cancer was accounted $32.1 \%$ of all cancer among females and the prevalence has increased from 2007 to 2011 [1].

Current treatments in breast cancer include surgery, chemotherapy, radiotherapy and hyperthermia as well hormone therapy. These treatments are usually combined, such as surgery with chemotherapy, radiotherapy with chemotherapy and hyperthermia with radiotherapy. Though these treatments are amenable, it is restricted by possible adverse effect due to artificial anticancer drugs such as diarrhoea, rash, mucosal inflammation, febrile neutropenia [2] and significant toxicities associated with this adjuvant therapy [3].

Revised Manuscript Received on June 22, 2019.

Hanim Saim, School of Biomedical Engineering \& Health Science, Faculty of Engineering, Universiti Teknologi Malaysia, 81310 Skudai, Johor.

Maheza Irna Mohamad Salim, School of Biomedical Engineering \& Health Science, Faculty of Engineering, Universiti Teknologi Malaysia, 81310 Skudai, Johor.

Khairunadwa Jemon, Department of Biosciences, Faculty of Science, Universiti Teknologi Malaysia, 81310 Skudai Johor
Curcumin is a hydrophobic polyphenol, a dietary phytochemical and a principle active ingredient derived from turmeric. It was first isolated by Vogel and Pelletier in 1815 , its chemical structure and synthesis confirmed by Lampe et al. in 1910 and 1913 [4]. Chemically, it is a bis$\alpha, \beta$ unsaturated $\beta$ - diketone named (E,E)-1,7-bis(4-hydroxy3-methoxyphenyl)-1,6-heptadiene-5dione or commonly called diferuloylmethane [5]. Commercial curcumin is a mixture of curcuminoids, containing approximately $77 \%$ diferuloylmethane (Curcumin I), 17\% demethoxycurcumin (Curcumin II), and 5\% bisdemethoxycurcumin (Curcumin III) [4].

Curcumin has chemopreventive and therapeutic activity against various breast cancer cell lines [6]-[8]. Besides, previous study of curcumin in vivo presented significant reduction of tumor multiplicity, prolongation of tumour-free survival inhibition of the tumor growth, reduced the tumor size and weight during the treatment [7]-[10].

Additionally, curcumin also used in combination with other modalities. The tumor growth in female CD1 nude mice suppressed by the combination treatment of curcumin $(200 \mathrm{mg} / \mathrm{kg} /$ day $)$ and epigallocethun gallate $(25 \mathrm{mg} / \mathrm{kg} /$ day $)$ for 10 weeks [11]. Further, study reported that combination of curcumin $(50 \mathrm{mg} / \mathrm{kg})$ and metformin $(80 \mathrm{mg} / \mathrm{kg})$ in 14 days showed the highest significant reduction in the tumour size [12]. While the combination of taxol with curcumin in the BT-474 xenograft model had an antitumour effect comparable with taxol and herceptin treatment [10].

On the other hand, hyperthermia is broadly referred as a condition where mean body temperature higher than normal. In medicine, hyperthermia therapy (HT) or thermotherapy is the procedure to elevate the temperature of a part of or the whole body above normal for a definite period of time, using external and internal heating device. Hyperthermia treatment temperatures in clinical procedure range between $39-45{ }^{\circ} \mathrm{C}$ [13]. Hyperthermia is commonly applied as combination with various established cancer treatment modalities such as radiotherapy [14], chemotherapy [15], surgery and immunotherapy [16]. The temperature and duration of HT damages cells and enhances radiotherapy and chemotherapy sensitivity [17].

The cellular changes such as protein denaturation and aggregation, inducing cell cycle interruption and apoptosis, increasing membrane permeability, altering $\mathrm{Ca} 2+$ homeostasis and promoting intracellular accumulation of chemical agents show the efficiency of hyperthermia in cancer treatment.

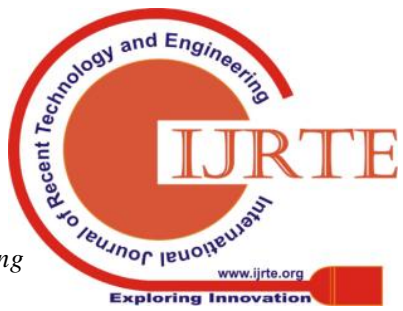


As well as, the cytotoxic effects of hyperthermia is including activation of the immune systems against tumor, enhancing drug delivery and improvement of oxygenation [18], [19]. This study aimed to investigate the efficacy of curcumin combined with fever range whole-body hyperthermia in suppressing breast cancer tumor growth.

\section{MATERIALS \& METHODOLOGY}

\section{Animals}

Animal care and use in this study was conducted according to standard ethical guidelines and experimental protocols approved by the Animal Ethics Committee of Universiti Kebangsaan Malaysia, approval number (UTM/2017/MAHEZA IRNA/22-NOV./884-NOV.-2017MARCH-2019).

Female BALB/c mice (6-8 weeks old) [20] with $17-25 \mathrm{~g}$ body weight was purchased from local university Animal Research Unit. Animals were housed in cage (max 5/cage) with 12-h dark and light cycles, fed standard laboratory diet and water ad libitum daily and bedding changed every two days. The mice allowed to acclimatize for at least 7 days before experiments.

\section{Selection of curcumin dose}

Dosage range between $50 \mathrm{ug} / \mathrm{kg}$ to $200 \mathrm{mg} / \mathrm{kg}$ were effectively inhibit breast cancer growth as reported in previous studies on various animal model species [11], [21], [22]. However, curcumin dosage of $50 \mathrm{mg} / \mathrm{kg}$ was used to $\mathrm{Balb} / \mathrm{c}$ mice induced EMT6 breast cancer tumor size [12]. Therefore this study is used as reference.

\section{Selection of temperature and duration of fever range whole-body hyperthermia (FRWBH) treatment}

This study was initiated by the optimization of temperature and duration of FRWBH treatment conducted using water-filtered infrared-A (wIRA) by Hydrosun Medizintechnik $\mathrm{GmbH}$ device to observe the viability of mice under certain temperature of mild hyperthermia within the range of $39.0-40.0^{\circ} \mathrm{C}$ which commonly used in clinical procedure [16].

\section{Tumor inoculation}

EMT6 mouse mammary tumor cells were maintained in Waymouth's Media MB752/1 Liquid Medium (1x) Thermo Fisher [23] supplemented with $10 \%$ heat-inactivated Fetal Bovine Serum (FBS) Thermo Fisher and 1\% antibiotics $(100 \mathrm{U} / \mathrm{ml}$ penicillin and $100 \mu \mathrm{g} / \mathrm{ml}$ streptomycin). Cells were harvested using trypsin solution TrypLE ${ }^{\mathrm{TM}}$ Express Enzyme (1x) without phenol red, washed, centrifuged and re-suspended in Phosphate Buffered Saline (PBS), at a density of $5 \times 10^{6}$ cells $/ \mathrm{ml}$ [20]. Cells count performed using haemocytometer plate and the cells re-suspended in PBS. Each BALB/c mouse received $5 \times 10^{5}$ EMT6 cells in $100 \mu l$ PBS [24] injected subcutaneously at right flank.

\section{FRWBH treatment}

Whole body Fever Range hyperthermia (FRWBH) was conducted twice using water-filtered infrared-A (wIRA) by Hydrosun Medizintechnik GmbH device on day-17 and day23 post inoculation. Mice were anesthetized with the mixture of ketamine $(150 \mathrm{mg} / \mathrm{kg})$ and xylazine $(7.5 \mathrm{mg} / \mathrm{kg})$ and hyperthermia treatment were conducted at $39^{\circ} \mathrm{C}( \pm 0.5)$ maintained for up to 15 minutes. Rectal temperature monitored continuously in each mouse using small animal rectal thermostat probes [25] along the treatment (Figure 1).

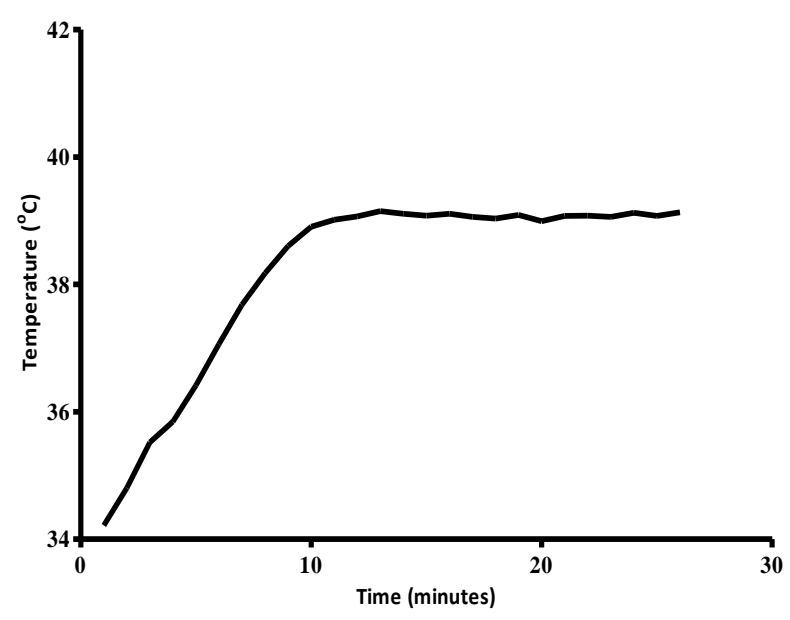

Fig. 1 Mice rectal temperature recorded during FRWBH treatment. Median rectal temperature of the mice during hyperthermia treatment was $39.05^{\circ} \mathrm{C}$ and mean rectal temperature of the mice was $38.17^{\circ} \mathrm{C}$. Approximately 12 minutes required for the rectal temperature to reach $39.0{ }^{\circ} \mathrm{C}(\mathbf{\pm 0 . 5})$.

\section{General toxicity assessment}

Mice body weight recorded every other day after inoculation as a general indicator of toxicity. Drug-specific toxicities such as liver toxicity, renal toxicity, neurotoxicity were not measured in this study.

\section{Antitumor activity assessment}

Mice were randomly distributed into four groups $(n=5$ for each group). Group 1 received no treatment (CONTROL). Group 2 received $50 \mathrm{mg} / \mathrm{kg}$ bwt curcumin orally (CUR) using oral gavage daily for 14 days starting day-14 post inoculation until day- 27 post inoculation. Group 3 received whole-body hyperthermia treatment conducted at $39^{\circ} \mathrm{C}$ $( \pm 0.5)$ maintained for up to 15 minutes on day-17 and day23 post inoculation (FRWBH). Group 4 received both curcumin $50 \mathrm{mg} / \mathrm{kg}$ bwt orally and hyperthermia treatment conducted at $39^{\circ} \mathrm{C}( \pm 0.5)$ maintained for up to 15 minutes on day-17 and day-23 post inoculation (CUR+FRWBH). Summary of mice group allocation and the treatment received as shown in Table 1 . Mice were sacrificed on day28 post inoculation. Tumor size was measured and recorded every other day using electronic caliper to determine the diameter [26]. Tumor volume was calculated according to the formula 'tumor volume $=1 / 2(\mathrm{ab} 2)$ ' in which ' $\mathrm{a}$ ' is the longest diameter and ' $b$ ' is the shortest diameter of the tumor. 
Table 1. Summary of mice group and the treatment received

\begin{tabular}{ll}
\multicolumn{1}{c}{ Group } & Treatment received \\
\hline CONTROL & No treatment received. \\
CUR & Orally $50 \mathrm{mg} / \mathrm{kg}$ daily (day-14 to day-27 post \\
& inoculation). \\
FRWBH & Whole-body hyperthermia treatment \\
& conducted at $39^{\circ} \mathrm{C}( \pm 0.5)$ maintained for up \\
& to 15 minutes on day-17 and day-23 post \\
& inoculation. \\
CUR + & Orally $50 \mathrm{mg} / \mathrm{kg}$ daily (day-14 to day-27 post \\
FRWBH & inoculation and Whole-body hyperthermia \\
& treatment conducted at $39^{\circ} \mathrm{C} \quad( \pm 0.5)$ \\
& maintained for up to 15 minutes on day-17 \\
& and day-23 post inoculation.
\end{tabular}

\section{Statistical Analysis}

Data analyzed using Graph Pad Prism Windows 5.00 and presented as mean \pm SEM (standard error of mean). Oneway analysis of variance (ANOVA) was used to measure variations between different groups. Unpaired-samples t-test was used to examine the effects of different treatments on the tumor size and body weight. The level of significance was set at $\mathrm{p}<0.05$.

\section{RESULTS}

Selection of temperature and duration of fever range whole-body hyperthermia (FRWBH) treatment

Each of experiment group for temperature optimization consist of two mice $(n=2)$. All mice $(n=2)$ were dead immediately after completion of FRWBH treatment of 40.0 ${ }^{\circ} \mathrm{C}( \pm 0.5)$ for 30 minutes. The second group of mice is exposed to FRWBH treatment of $40.0{ }^{\circ} \mathrm{C}( \pm 0.5)$ for 15 minutes. One mouse $(n=1)$ dead a day after the treatment and another one $(n=1)$ survived up to 14 days after the treatment. The result was similar for mice received FRWBH treatment of $39.0{ }^{\circ} \mathrm{C}( \pm 0.5)$ for 30 minutes. However, all mice $(\mathrm{n}=2)$ which received FRWBH treatment of $39.0^{\circ} \mathrm{C}( \pm$ $0.5)$ for 15 minutes were survived more than 10 days after the treatment. Therefore, the temperature of $39.0^{\circ} \mathrm{C}( \pm 0.5)$ was selected as optimum temperature and duration of 15 minutes as duration method for FRWBH treatment of this study.

\section{General toxicity assessment}

Table 2 shows the result for mice body weight for each group. Mice body weights recorded every other day as an indicator of general health and treatment-induced toxicity. Overall result shows there is significant different of mean body weight among the groups $(\mathrm{p}<0.0001)$ and weight gained observed significantly in all groups within 28 days of experiment.
Table. 2 Effect of $50 \mathrm{mg} / \mathrm{kg}$ curcumin, FRWBH and combination of both on mice body weight.

\begin{tabular}{ccccc} 
Group & $\begin{array}{c}\text { Mean body } \\
\text { weight }(\mathrm{g})\end{array}$ & $p$ value & $\begin{array}{c}\text { Body weight } \\
\text { gain (g) }\end{array}$ & $\begin{array}{c}p \\
\text { value }\end{array}$ \\
\hline CONTROL & $24.21 \pm 0.14$ & - & $1.21 \pm 0.14$ & - \\
\hline CUR & $24.53 \pm 0.16$ & 0.1443 & $1.13 \pm 0.16$ & 0.5567 \\
\hline FRWBH & $23.96 \pm 0.13$ & 0.1975 & $0.36 \pm 0.13$ & 0.0004 \\
CUR + & $26.64 \pm 0.13$ & $<0.0001$ & $0.64 \pm 0.13$ & 0.0089 \\
FRWBH & & & & \\
\hline
\end{tabular}

\section{Antitumor activity assessment}

Tumor observed to continuous grow starting on day-7 and the time course for tumor volume changes after the initiation of treatment on day-14. Figure 2 shows results of the tumor growth profile for all groups during experiment. Mean tumor volume of the mice after 28 days was significantly different among groups $(\mathrm{p}=0.0042, \mathrm{p}<0.01)$. Mice treated with CUR+FRWBH proved significantly delayed tumor growth as compared to curcumin and control. The tumor in control group grew progressively as compared to others group, while FRWBH demonstrated the greater tumor growth control among the group.

Tumor inhibition rate for all treatment groups is shows in Figure 3. CUR+FRWBH inhibition rate is higher than curcumin with $68.45 \%$ and FRWBH has the highest inhibition rate.

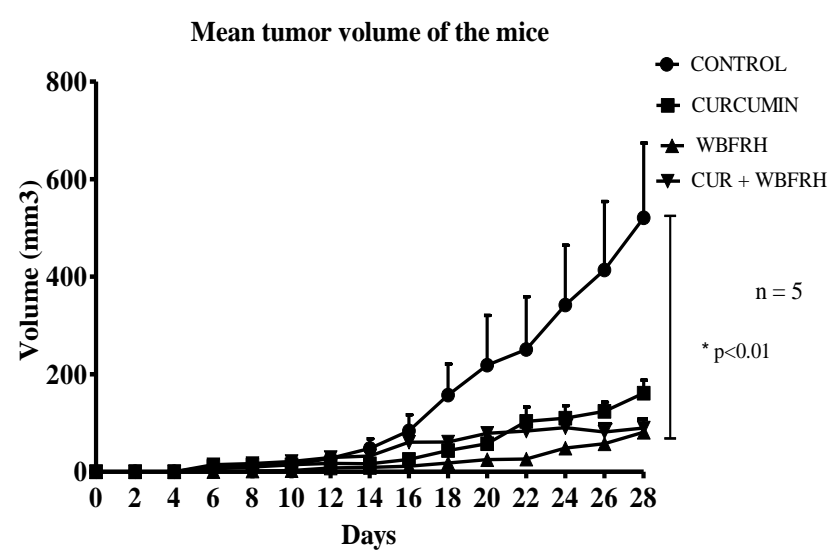

Fig. 2 Antitumor effect of curcumin combined with FRWBH in breast cancer-induced Balb/c mice. Mice injected subcutaneously with EMT6 cells and tumor was allowed to grow for 14 days before initiation of treatment. Values are the mean \pm SEM of 5 mice. ANOVA $p<0.01, p<0.05$ compared with control, $p<0.05$ compared to FRWBH alone. 


\section{Tumor growth inhibition (TGI\%)}

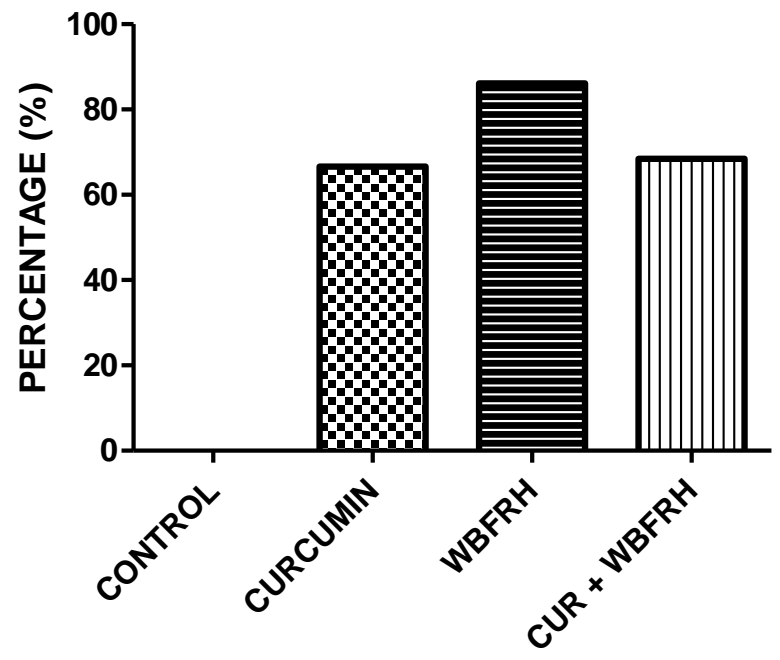

Fig. 3 Tumor growth inhibition rate of treatment compared to control.

\section{DISCUSSION}

Breast cancer is one of the main cancer among female population worldwide. Due to the elevated death rate related with cancer and severe side effects of chemotherapy and radiation therapy, many cancer patients seek alternative complementary and alternative medicines (CAM) as method of treatment. CAM have related with natural herbal medicines and herbal plant, which are alleged to various biological and molecular mechanisms by inhibiting the growth of cancer. Hyperthermia is commonly applied as combination with various established cancer treatment modalities such as radiotherapy, chemotherapy, surgery and immunotherapy, effectively increased antitumor effect. Thus, this study is conducted to search for new alternative treatment of breast cancer by funds of curcumin combined with hyperthermia treatment.

In this study, each group received single treatment of curcumin or hyperthermia and combination of both to see the efficacy of each treatment towards the tumor growth and animal health. Mean body weight of treated mice were higher than control mice and there were weight gained in all groups up to 28 days of experiment that may due to tumor growth. It is better than the decline in body weight due to toxicity [27]. General observation of animal health showed that the treatments were well tolerated by the mice and nontoxic to the mice.

The results of our study towards tumor volume of mice suggested that FRWBH was effectively reduced tumor size with the highest inhibition rate $(86.14 \%)$. The findings are equivalent with previous studies which stated hyperthermia was cytotoxic to breast cancer cells [30], [31]. However, there was rapid tumor growth few days before experiment ended which reinforced that hyperthermia as singletreatment modality had low response rate and short duration limits [16], [32].

Even though the tumor inhibition rate in combined group was lower than FRWBH group, our results show the tumor growth in CUR+FRWBH group was plateau few days before end of experiment compared to others treatment group, indicating the combination treatment had better effect on tumor growth suppression compared to CUR and FRWBH treatments. The findings are in line with previous studies that combination of radiotherapy (RT), chemotherapy (CT) or RT plus CT treatment with hyperthermia were better, compared with the same treatment without hyperthermia in mice and human [13], [25], [33].

In summary, we demonstrated that FRWBH combined with curcumin was beneficial against solid tumor than treatment with FRWBH or curcumin alone, with respect to inhibition of tumor growth without general toxicity effect.

\section{CONCLUSION}

As from this perspective, we suggest that combination treatment with FRWBH and curcumin is actually useful as alternative antitumor treatment for breast cancer. However, further study with larger sample and various temperature and duration of whole body fever range hyperthermia should be conducted in the future in regards to explore the mechanism of action of this combination treatment.

\section{ACKNOWLEDGMENT}

The authors'work was supported by grants from Ministry of Higher Education for Universiti Teknologi Malaysia (KPT-UTM RUG grant 4F274 15H87).

\section{REFERENCES}

1. M. Azizah, I. T. Nor Saleha, A. Noor Hashimah, Z. A. Asmah, and W. Mastulu, "Malaysian National Cancer Registry Report 2007-2011, Malaysia Cancer Statistics, Data and Figure," Natl. Cancer Inst., vol. 16, p. 203, 2016.

2. E. D. Pisano et al., "New England Journal," pp. 1773-1784, 2005.

3. E. A. Rakha et al., "Tubular carcinoma of the breast: Further evidence to support its excellent prognosis," J. Clin. Oncol, vol. 28, no. 1, pp 99-104, 2010

4. H. Zhou, C. S. Beevers, and S. Huang, "The targets of curcumin," Curr. Drug Targets, vol. 12, no. 3, pp. 332-47, 2011

5. A. Goel, A. B. Kunnumakkara, and B. B. Aggarwal, "Curcumin as 'Curecumin': From kitchen to clinic," Biochem. Pharmacol, vol. 75, no. 4, pp. 787-809, 2008.

6. S. Bimonte et al., "Dissecting the Role of Curcumin in Tumour Growth and Angiogenesis in Mouse Model of Human Breast Cancer," vol. 2015, pp. 16-20, 2015.

7. L. Masuelli et al., "Curcumin induces apoptosis in breast cancer cell lines and delays the growth of mammary tumors in neu transgenic mice," J. Biol. Regul. Homeost. Agents, vol. 27, no. 1, pp. 105-119, 2013.

8. L. Lv et al., "Enhancing curcumin anticancer efficacy through di-block copolymer micelle encapsulation," J. Biomed. Nanotechnol, vol. 10, no. 2, pp. 179-193, 2014.

9. E. Bachmeier et al., "The chemopreventive polyphenol curcumin prevents hematogenous breast cancer metastases in immunodeficient mice," Cell. Physiol. Biochem., vol. 19, no. 1-4, pp. 137-152, 2007.

10. H.-W. Lai et al., "The Potential Utility of Curcumin in the Treatment of HER-2-Overexpressed Breast Cancer: An In Vitro and In Vivo Comparison Study with Herceptin," Evidence-Based Complement. Altern. Med., vol. 2012, pp. 1-12, 2012.

11. T. J. Somers-Edgar, M. J. Scandlyn, E. C. Stuart, M. J. Le Nedelec, S. P. Valentine, and R. J. Rosengren, "The combination of epigallocatechin gallate and curcumin suppresses ER??-breast cancer cell growth in vitro and in vivo," Int. J. Cancer, vol. 122, no. 9, pp 1966-1971, 2008

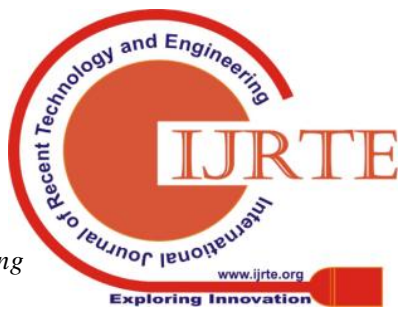


12. R. R. Falah, W. H. Talib, and S. J. Shbailat, "Combination of metformin and curcumin targets breast cancer in mice by angiogenesis inhibition, immune system modulation and induction of p53 independent apoptosis," Ther. Adv. Med. Oncol., vol. 9, no. 4, pp. 235-252, 2017.

13. A.Bettaieb, P. K. Wrzal, and D. A. Averill-bates, "Hyperthermia: Cancer Treatment and Beyond," Cancer Treat. Innov. Approaches. B. 2, pp. 257-283, 2013.

14. N. R. Datta et al., "Local hyperthermia combined with radiotherapy and-/or chemotherapy: Recent advances and promises for the future," Cancer Treat. Rev., vol. 41, no. 9, pp. 742-753, 2015.

15. G. Hegyi, G. P. Szigeti, and A. Szász, "Hyperthermia versus oncothermia: Cellular effects in complementary cancer therapy," Evidence-based Complement. Altern. Med., vol. 2013, 2013.

16. Z. Behrouzkia, Z. Joveini, B. Keshavarzi, N. Eyvazzadeh, and R. Z. Aghdam, "Hyperthermia: How can it be used?," Oman Med. J., vol. 31, no. 2, pp. 89-97, 2016.

17. M. Mallory, E. Gogineni, G. C. Jones, L. Greer, and C. B. Simone, "Therapeutic hyperthermia: The old, the new, and the upcoming," Crit. Rev. Oncol. Hematol., vol. 97, pp. 56-64, 2015.

18. J. R. Ostberg, C. Gellin, R. Patel, and E. A. Repasky, "Regulatory potential of fever-range whole body hyperthermia on Langerhans cells and lymphocytes in an antigen-dependent cellular immune response," J. Immunol., vol. 167, pp. 2666-2670, 2001.

19. R. D. Issels, "Hyperthermia adds to chemotherapy," Eur. J. Cancer, vol. 44, no. 17, pp. 2546-2554, 2008.

20. W. H. Talib and S. Saleh, "Propionibacterium acnes augments antitumor, anti-angiogenesis and immunomodulatory effects of melatonin on breast cancer implanted in mice," PLoS One, vol. 10, no. 4, pp. 1-13, 2015.

21. Q. Zhou, X. Wang, X. Liu, H. Zhang, Y. Lu, and S. Su, "Curcumin enhanced antiproliferative effect of mitomycin $\mathrm{C}$ in human breast cancer MCF-7 cells in vitro and in vivo," Acta Pharmacol. Sin, vol. 32, no. 11 , pp. 1402-1410, 2011.

22. Z.-D. Lv et al., "Curcumin induces apoptosis in breast cancer cells and inhibits tumor growth in vitro and in vivo," Int. J. Clin. Exp. Pathol., vol. 7, no. 6, pp. 2818-24, 2014.

23. S. Rockwell et al., "Preclinical evaluation of Laromustine for use in combination with radiation therapy in the treatment of solid tumors," Int. J. Radiat. Biol., vol. 88, no. 3, pp. 277-285, 2012.

24. R. M. Gorczynski et al., "Breast cancer cell CD200 expression regulates immune response to EMT6 tumor cells in mice," Breast Cancer Res. Treat., vol. 123, no. 2, pp. 405-415, 2010.

25. R. W. Rowe et al., "Fever-range whole body thermotherapy combined with oxaliplatin: a curative regimen in a pre-clinical breast cancer model," Int. J. Hyperthermia, vol. 26, no. 6, pp. 565-76, 2010.

26. K. Sumiyoshi, F. R. Strebel, R. W. Rowe, and J. M. C. Bull, "The effect of whole-body hyperthermia combined with 'metronomic' chemotherapy on rat mammary adenocarcinoma metastases 3150 ," Int.J.Hyperthermia, vol. Internatio, pp. 2-118, 2003.

27. J. M. C. Bull, F. R. Strebel, G. N. Jenkins, W. Deng, and R. W. Rowe, "The importance of schedule in whole body thermochemotherapy.," Int. J. Hyperthermia, vol. 24, no. 2, pp. 171-81, 2008.

28. Farhangi et al., "Protective effects of dendrosomal curcumin on an animal metastatic breast tumor," Eur. J. Pharmacol., 2015.

29. S. Shiri et al., "Dendrosomal curcumin suppresses metastatic breast cancer in mice by changing M1/M2 macrophage balance in the tumor microenvironment," Asian Pacific J. Cancer Prev., vol. 16, no. 9, pp. 3917-3922, 2015.

30. H. Lee et al., "Response of breast cancer cells and cancer stem cells to metformin and hyperthermia alone or combined," PLoS One, vol. 9, no. 2, p. e87979, 2014

31. S. Kossatz et al., "Efficient treatment of breast cancer xenografts with multifunctionalized iron oxide nanoparticles combining magnetic hyperthermia and anti-cancer drug delivery." Breast Cancer Res., vol. 17 , p. 66, 2015.

32. H. I. Robins, "Role of Whole-Body Hyperthermia in the Treatment of Neoplastic Disease: Its Current Status and Future Prospects Role of Whole-Body Hyperthermia in the Treatment of Neoplastia Disease : Its Current Status and Future Prospects1," Cancer Res., vol. 44, no. April 1982, pp. 4878-4883, 1984

33. M. Hurwitz and P. Stauffer, "Hyperthermia, radiation and chemotherapy: The role of heat in multidisciplinary cancer care," Semin. Oncol, vol. 41, no. 6, pp. 714-729, 2014. 